\title{
CORRIGENDA
}

\section{Knockdown of angiotensinogen by shRNA-mediated RNA interference inhibits human visceral preadipocytes differentiation}

Z-W Ye ${ }^{1,2}, \mathrm{X}-\mathrm{M} \mathrm{Wu}^{2}$, L-J Zhang ${ }^{1}$, Z-L Huang ${ }^{1}$ and J-G Jiang ${ }^{2}$

${ }^{1}$ School of Applied Chemistry and Biological Technology, Shenzhen Polytechnic, Shenzhen, China and ${ }^{2}$ College of Food and Bioengineering, South China University of Technology, Guangzhou, China

Correspondence: Dr L-J Zhang or Dr Z-L Huang, School of Applied Chemistry and Biological Technology, Shenzhen Polytechnic, Shenzhen, Guangdong 518055, China. E-mail: c7zlj@szpt.edu.cn (L-JZ) or hzl@szpt.edu.cn (Z-LH); or Professor J-G Jiang, College of Food and Bioengineering, South China University of Technology, Guangzhou 510640, China. E-mail: jgjiang@scut.edu.cn

Correction to: International Journal of Obesity (2010) 34, 157-164; doi:10.1038/ijo.2009.197; published online 29 September 2009.

The authors would like to apologize for the omission of two authors, Dr Li-Jun Zhang and Dr Zhi-Li Huang, from this article. The corrected author list and affiliations are shown above.

\section{ACKNOWLEDGEMENTS}

This work was supported by Science and Technology Project of Shenzhen City (07KJba160).

\section{Public health strategy against overweight and obesity in Mexico's National Agreement for Nutritional Health}

L Latinovic and L Rodriguez Cabrera

International Journal of Obesity (2013) 37, 1616; doi:10.1038/ijo.2013.186

Correction to: International Journal of Obesity Supplements (2013) 3, S12-S14; doi:10.1038/ijosup.2013.5

Since the publication of this paper, it has been noticed that the author name L Latnovic has been listed incorrectly. The correct spelling is L Latinovic.
The authors would like to apologize for the error. 